\title{
Análisis de sectores clave de la economía española a partir de la Matriz de Contabilidad Social de España 2007*
}

\author{
José Manuel Cansino Muñoz-Repiso a , Manuel Alejandro Cardenete

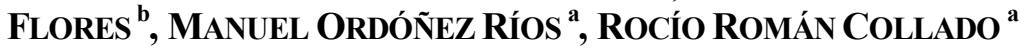 \\ a Universidad de Sevilla, Facultad CC.EE., Avda. Ramón y Cajal, 1, 41018 Sevilla, España. E- \\ mail:jmcansino@us.es, manuelor@us.es, rroman@us.es \\ ${ }^{b}$ Universidad Pablo de Olavide, Facultad CC.EE., Ctra. Utrera Km.1, s/n, 41013 Sevilla, España. \\ E-mail:macardenete@uloyola.es
}

\begin{abstract}
RESUMEN
Las matrices de Contabilidad Social constituyen una importante base de datos que proporcionan información relevante sobre la estructura económica y social de un área para un determinado período de tiempo. Esta información permite realizar un análisis integral de la estructura productiva de dicha área. En este artículo se presenta la Matriz de Contabilidad Social (MCS) para España correspondiente al año 2007 a precios básicos. A partir de ésta se identifican los sectores clave de la economía española a través de tres metodologías diferentes: la metodología propuesta por Rasmussen, el método de extracción hipotética y, finalmente, el método de la Matriz del Producto Multiplicador. El análisis de los sectores clave con el uso de estas tres metodologías da lugar a conclusiones que, en algunos casos, son muy diferentes. Por otro lado, los resultados se enriquecen al introducir como endógenas las cuentas de Trabajo, Capital y Consumo en el modelo.
\end{abstract}

Palabras clave: Matriz de Contabilidad Social, Tablas Input-Output, sectores clave.

\section{Key Sectors Analysis of the Spanish Economy Using a Social Accounting Matrix for 2007}

\begin{abstract}
Social Accounting Matrices are important databases that provide relevant information about the economic and social structure of an area for a period of time. This information allows researchers to develop an integral analysis of the productive structure of that area. This article presents the Social Accounting Matrix (SAM) for the Spanish economy for 2007 at basic prices. From SAM for Spain the key sectors of the Spanish economy are identified across three different methodologies: the methodology developed by Rasmussen, the hypothetical extraction method and, finally, using the technique of the Multiplier Product Matrix. The key sectors analysis using these three different methodologies lead to conclusions that, in some cases, are very different. In addition, the results improve when labor income, capital income, private and consumption are included as endogenous accounts in the model.
\end{abstract}

Keywords: Social Accounting Matrix, Input-Output Tables, Key sectors.

JEL Classification: C67, D57

\footnotetext{
* Cansino, Ordóñez y Román agradecen la financiación procedente del grupo de investigación SEJ-132 de la Consejería de Innovación de la Junta de Andalucía. Cansino y Román agradecen también el apoyo recibido de la Cátedra de Economía de la Energía y del Medio Ambiente de la Universidad de Sevilla-Fundació Roger Torné. Cardenete agradece la financiación procedente de los proyectos MICINN-ECO200911857, SGR2009-5781 y SEJ479. Los autores agradecen los comentarios y sugerencias de dos revisores anónimos aunque la responsabilidad del articulo es únicamente nuestra.
}

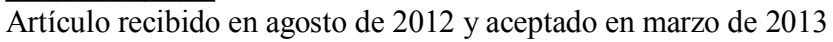

Artículo disponible en versión electrónica en la página www.revista-eea.net, ref. ə-31205 


\section{INTRODUCCIÓN}

Las matrices de Contabilidad Social (MCS), al igual que las Tablas InputOutput (TIO), permiten representar el conjunto de transacciones realizadas en una economía durante un determinado período de tiempo. Sin embargo, las TIO no abarcan la totalidad de dichas transacciones, ya que se limitan a las interacciones entre los diferentes sectores productivos y, a las de éstos, con los factores primarios y con los agentes económicos a través de la demanda final que realizan. Así pues, las MCS amplían esta información al incluir las relaciones que aparecen entre los inputs primarios y la demanda final quedando reflejadas todas ellas en el flujo circular de la renta. Las MCS no sólo permiten describir las operaciones de producción, distribución, acumulación y usos de la renta, tanto dentro de la propia economía como con el resto del mundo, sino que también resultan de gran utilidad para el análisis económico. Las MCS incluyen una importante base de datos que va a servir de soporte estadístico para la construcción de modelos económicos con los que analizar los efectos asociados a cualquier shock $\mathrm{o}$ al resultado de diferentes políticas económicas.

El primer objetivo de este artículo es construir una MCS para España correspondiente al año 2007 a precios básicos ${ }^{1}$, que sirva de herramienta básica para identificar los sectores clave de la economía española. La justificación para elegir el año 2007 es la disponibilidad de información estadística, ya que es el año más reciente del Marco Input-Output (I-O) de que dispone el Instituto Nacional de Estadística (INE) en el momento de realizar esta investigación. Esta información, junto con los cuadros contables que para el mismo año ha elaborado dicho Instituto, es la fuente de datos que ha sido utilizada para la construcción de la MCS para España.

El segundo objetivo del artículo es identificar los sectores clave de la economía española en 2007. Los sectores clave son aquellos que tienen una mayor potencialidad para estimular la producción de otros sectores y, por tanto, el crecimiento de los ingresos y el empleo (Rasmussen, 1956). Este proceso se debe a que una parte de la demanda estimula a otros sectores para satisfacer sus necesidades de inputs intermedios y, por otra, la oferta estimula la producción ya que ésta puede inducir para ser utilizada como insumo en otras actividades. Esta interdependencia sectorial denominada eslabonamientos (Hirschman, 1958), puede identificarse y cuantificarse mediante varios métodos. Este artículo utiliza tres. Primero, la metodología utilizada por Rasmussen (1956) cuya base son los multiplicadores simples para el caso de la TIO, o multiplicadores contables en el caso de la MCS mediante la endogeneización de cuentas. Segundo, la meto-

\footnotetext{
${ }^{1}$ La diferencia esencial entre una MCS a precios básicos y una MCS a precios de adquisición está en que la segunda se incluyen los impuestos indirectos netos sobre los productos y los márgenes comerciales y de transporte.
} 
dología de extracción hipotética de sectores propuesta por Dietzenbacher et al. (1993) que cuantifica la disminución en la producción total que tendría la eliminación hipotética de un determinado sector económico. Tercero, la elaboración de la Matriz Producto Multiplicador que tiene en cuenta de una manera conjunta el efecto difusión y el efecto arrastre de los sectores económicos (Sonis et al, 1997). Sin embargo, la aplicación de estos métodos no da lugar a resultados idénticos sino que se aprecian diferencias. Entre los trabajos que han aplicado estas metodologías para el caso de España en al ámbito nacional o regional se pueden citar, entre otros, Dietzenbacher et al. (2005), Cardenete y Sancho (2006), Robles y Sanjuán (2008), Llano (2009), Cardenete et al. (2008, 2010), Alcántara et al. (2010), Guerra y Sancho (2010) y Cámara et al. (2011).

Este artículo ha sido estructurado en siete apartados. Tras esta introducción, el apartado segundo muestra las bases de datos utilizadas y la metodología empleada para su elaboración, así como la estructura de la MCS para España de 2007 a precios básicos. El tercero, cuarto y quinto apartados se dedican al análisis sectorial a través de la metodología de Rasmussen (1956), del método de extracción hipotética y de la Matriz Producto Multiplicador (MPM), respectivamente. Un análisis comparativo de los resultados se realiza en el sexto apartado. El apartado final presenta las conclusiones.

\section{FUENTES DE DATOS Y METODOLOGÍA}

\subsection{Estructura}

Las matrices de Contabilidad Social tienen por finalidad la representación del conjunto de todas las transacciones realizadas en una economía durante un determinado período de tiempo. Cada MCS es una base de datos muy rica organizada en forma de tabla de doble entrada donde se recoge la información económica y social correspondiente a las transacciones habidas entre todos los agentes económicos. Una MCS amplía la información contenida en una TIO ya que, además de incluir a ésta, incluye todos los flujos entre los inputs primarios y la demanda final. Por tanto, en una MCS queda reflejado el flujo circular de la renta de una economía.

El uso de las MCS fue iniciado por Stone (1962) que publicó la primera para el Reino Unido. Sin embargo, dada su utilidad para conocer las relaciones intersectoriales de la economía y la distribución de la renta, las primeras MCS fueron elaboradas para países en desarrollo con la finalidad de poner en marcha programas que permitiesen reducir la pobreza ${ }^{2}$.

En España, la primera MCS fue realizada por Kehoe et al. (1988) para el año

\footnotetext{
${ }^{2}$ Entre otras, hay que destacar la MCS de Sri Lanka elaborada por Pyatt y Roe (1977) por el impulso que se dio en este campo.
} 
1980, utilizándose, posteriormente, como soporte numérico de un Modelo de Equilibrio General para estudiar los efectos de la introducción del Impuesto sobre el Valor Añadido en la economía española ${ }^{3}$. Más recientemente Cardenete y Sancho (2007) elaboraron una MCS para la economía española para el año 1995 a precios de adquisición en lugar de a precios básicos, partiendo del nuevo marco contable del Sistema Europeo de Cuentas Integradas 1995 (SEC95) y siguiendo la metodología de entropía cruzada ${ }^{4}$. Rodríguez y Llanes (2005) utilizan esta metodología para estimar anualmente MCS.

Las MCS tienen dos grandes finalidades. La primera es de tipo descriptiva, esto es, se trata de una importante base de datos que proporciona información sobre la estructura económica y social de una economía en un determinado período de tiempo. La segunda finalidad es de tipo analítica, ya que una MCS sirve de soporte estadístico necesario para la construcción de modelos con los que analizar los efectos que generan la aplicación de determinadas políticas económicas. Los modelos que utilizan la MCS como base de datos se diferencian en dos grupos: los modelos lineales y los Modelos de Equilibrio General Aplicado (MEGA). Los primeros tienen una estructura formal similar al modelo de Leontief (1966). Los segundos son modelos de ecuaciones simultáneas que reflejan las condiciones de equilibrio de una economía. Estos modelos son más flexibles que los modelos lineales ya que no imponen tantas restricciones sobre el comportamiento de los agentes económicos y es posible formular relaciones de carácter no lineal (Cardenete y Llop, 2005).

Para la construcción de la MCS para España del año $2007^{5}$, las fuentes de datos utilizadas proceden del Marco I-O de España junto con los cuadros contables que para el mismo año ha elaborado el INE. La estructura de una MCS es flexible permitiendo diferentes formas de construcción al poderse desagregar las cuentas y los sectores que la forman ${ }^{6}$. El grado de desagregación suele depender

\footnotetext{
${ }^{3}$ Posteriormente se elaboraron otras MCS entre las que podrían destacarse la realizada por Uriel et al.(1997) en colaboración con el Instituto Nacional de Estadística (INE) y el Instituto Valenciano de Investigaciones Económicas (IVIE) para 1990, con un importante grado de desagregación de los hogares y de los factores respecto de las anteriores MCS. Con posterioridad se publicó una alternativa a esta matriz cuadrada con 82 instituciones por parte de Fernández y Polo (2001).

${ }^{4}$ La metodología de entropía cruzada es útil para la actualización de matrices y utiliza un procedimiento de ajuste basado en la resolución de un programa de optimización no lineal con restricciones. El objetivo es encontrar una nueva matriz obtenida a partir de otra inicial de tal manera que la variación entre los valores iniciales y los valores estimados sean mínimos y que, además, respeten las restricciones dadas, como los valores totales de las filas y columnas así como otras restricciones adicionales.

${ }^{5}$ La SAMESP07 se encuentra a disposición de los interesados que la soliciten.

${ }^{6}$ Como señala el SEC95 “... se pueden seleccionar tipos de agentes o grupos de agentes diferentes en cada cuenta sin perder por ello la coherencia y la integración del sistema de cuentas en su conjunto". Comisión Europea (1996:318).
} 
del objetivo del estudio y de la disponibilidad de los datos. La estructura de la MCS para España del año 2007 (SAMESP07) puede asimilarse, por ejemplo, a la incluida en Cardenete et al. (2008).

Para elaborar una MCS se necesitan cuatro submatrices, la Matriz de Consumos Intermedios, la Matriz de Factores Primarios, la Matriz de Demanda Final y la Matriz de Cierre. En esta MCS están contempladas 43 cuentas: 26 actividades productivas, dos factores productivos, Trabajo (27) y Capital (28), la Formación Bruta de Capital (31) para indicar el Ahorro/Inversión, dos cuentas para representar el consumo de los Hogares (29) y de las instituciones sin fines de lucro al servicios de los hogares (30). Para las Administraciones Públicas se incluyen las Cotizaciones Sociales que pagan los empleadores (37), los impuestos netos de subvenciones que recaen sobre los productos (39), otros impuestos indirectos netos de subvenciones sobre la producción (39) y el gasto de las AAPP (42). El sector exterior está reflejado en las importaciones/exportaciones (43). Para representar otras transferencias de renta entre los sectores institucionales se incluyen las sociedades no financieras (32), las instituciones financieras (33), rentas de la propiedad (34), prestaciones sociales (35), otras transferencias corrientes (36), cotizaciones sociales (38) e impuestos sobre la renta y otros (41).

\subsection{Matriz de Consumos Intermedios (MCI)}

La MCI está formada, tanto en filas como en columnas, por los sectores productivos. En el caso de la SAMESP07, las 75 ramas de actividad y/o 118 productos que aparecen en las tablas de Origen y de Destino se han agregado en 26 sectores. La correspondencia de productos y de ramas de actividad totales con los sectores productivos considerados en la SAMESP07 se describe en la Tabla 1.

Tabla 1

Estructura sectorial de la SAMESP07 y correspondencias con las ramas de actividad del Marco I-O de España 2007

\begin{tabular}{|l|c|}
\hline \multicolumn{1}{|c|}{ SAMESP07 } & Ramas de actividad \\
\hline 1. Agricultura, Ganadería y Silvicultura & 1,2 \\
\hline 2. Pesca & 3 \\
\hline 3. Carbón & 4 \\
\hline 4. Petróleo y gas natural & 5 \\
\hline 5. Extractivas no energéticas & 6,7 \\
\hline 6. Refino de petróleo & 8 \\
\hline 7. Energía eléctrica & 9 \\
\hline 8. Gas & 10 \\
\hline 9. Agua & 11 \\
\hline
\end{tabular}


Tabla 1 (continuación)

Estructura sectorial de la SAMESP07 y correspondencias con las ramas de actividad del Marco I-O de España 2007

\begin{tabular}{|l|c|}
\hline \multicolumn{1}{|c|}{ SAMESP07 } & Ramas de actividad \\
\hline 10. Alimentación, bebidas y tabaco & 12 a 16 \\
\hline 11. Textil y piel & 17 a 19 \\
\hline 12. Elaborados de madera & 20,21 \\
\hline 13. Industria química & 23 \\
\hline 14. Materiales de construcción & 25 a 28 \\
\hline 15. Metalurgia & 29 \\
\hline 16. Elaborados metálicos & 30 \\
\hline 17. Maquinaria & 31 a 35 \\
\hline 18. Vehículos & 36 \\
\hline 19. Elementos de transporte & 37 \\
\hline 20. Otras manufacturas & $22,24,38,39$ \\
\hline 21. Construcción & 40 \\
\hline 22. Comercio y restauración & 41 a 45 \\
\hline 23. Transporte y Comunicaciones & 46 a 52 \\
\hline 24. Otros servicios & 53 a 55,58 a 60 \\
\hline 25. Servicios destinados a la venta & $56,57,62,64,67,69,71,74$ \\
\hline 26. Servicios no destinados a la venta & $66,68,70,72,73,75$ \\
\hline
\end{tabular}

Fuente: Elaboración propia.

A partir de las tablas de Origen y Destino es posible obtener utilizando diferentes procedimientos, la matriz de coeficientes técnicos y, a partir de ésta, la tabla simétrica. Sin embargo, su elaboración no es fácil debido entre otros factores a la existencia de productos secundarios. Algunos modelos de transformación de las Tablas de Origen y Destino en Tablas I-O simétricas pueden verse en Eurostat (2008). También ten Raa y Rueda-Cantuche (2003) realizan una descripción completa de diferentes tratamientos de las producciones secundarias. Aunque desde el punto de vista metodológico, para Jansen y ten Raa (1990) el método de tecnología por producto es teóricamente superior al resto, presenta una serie de limitaciones, entre ellas, la aparición de coeficientes técnicos negativos y la necesidad de que el número de productos sea igual al número de sectores, esto es, que tanto las Tablas de Origen como de Destino sean cuadradas. Por este motivo, en nuestro caso, se ha elegido el modelo D, según la terminología de Eurostat (2008). Este modelo asume una estructura fija de venta de los productos, esto es, cada producto tiene su propia estructura de ventas con independencia del sector o rama de actividad donde haya sido producido. Se trata de un método muy extendido pues permite eliminar los dos inconvenientes que presenta el método de tecnología por producto, esto es, la negatividad de los 
coeficientes técnicos y la necesidad de que el número de productos no tenga que coincidir con el número de sectores.

Para el cálculo de la MCI se procede de la siguiente forma. Sea $V$ la Tabla de Origen a precios básicos, donde $v_{i, j}$ es la cantidad de producto $i$ consumido por el sector $j$, expresado en unidades monetarias; y sea $U$, la Tabla de Destino a precios básicos, donde $u_{i, j}$ es la cantidad (expresada en unidades monetarias) de producto $j$ producido por el sector $i$. Denotaremos por $e$, un vector de unos, $\operatorname{con}^{-1}$ indicaremos que se trata de la matriz inversa $\mathrm{y}$, por ${ }^{T}$, la matriz traspuesta. Por otro lado, el acento circunflejo^ nos indicará diagonalización.

Así, la matriz de coeficientes técnicos $A=a_{i, j}$, que nos indica la cantidad de producto del sector $i$ necesaria para la producción de una unidad de producto elaborado por el sector $j$, se podrá calcular a partir de la siguiente expresión:

$$
A=V^{T} \cdot(\widehat{V \cdot e})^{-1} \cdot U \cdot\left(V^{T} \cdot e\right)^{-1}
$$

Una vez obtenida la matriz de los coeficientes técnicos ${ }^{7}$, se procede a calcular la tabla simétrica o MCI mediante la siguiente expresión:

$$
C I=A \cdot\left(\widehat{V^{T} \cdot e}\right)
$$

\subsection{Matriz de Factores Primarios (MFP)}

En la MFP quedan reflejados los factores productivos empleados por cada sector. El resto de cuentas tienen valor cero. En ellas aparecen las cuentas de Trabajo (27) y Capital (28) que reflejan los sueldos y los salarios brutos y el Excedente Bruto de Explotación/ Renta Mixta, respectivamente. En esta matriz se incluyen también las cotizaciones sociales que pagan los empleadores (37), los impuestos netos de subvenciones sobre los productos (39) y sobre la producción (40). Los datos se obtienen directamente de la Tabla de Destino del Marco I-O. La última cuenta que aparece en esta matriz son las importaciones (43), que se obtiene a partir de la Tabla de Origen a precios básicos. Dichos datos se integran utilizando la siguiente expresión:

$$
M=V^{T} \cdot(\widehat{V . e})^{-1} \cdot m
$$

donde $M$ son la importaciones que incorporaremos a la MFP y $m$, son las importaciones recogidas de la Tabla de Origen.

\footnotetext{
${ }^{7}$ Esta matriz cumple el axioma F o de equilibrio financiero definido por Jansen y ten Raa (1990), según la cual los consumos intermedios de los diferentes sectores tienen que ser iguales a los valores consumidos y observados en la tabla de destino, denotándose por:
}

$$
e^{t} \cdot A \cdot\left(\widehat{V^{t}} \cdot e\right)=e^{t} \cdot U
$$




\subsection{Matriz de Demanda Final (MDF)}

La MDF detalla los usos finales de los bienes y servicios producidos por los diferentes sectores productivos: el consumo de los hogares (29), el consumo de las ISFLSH (30), la Formación Bruta de Capital (31), las exportaciones (43), y el consumo de las AAPP (42). Todos los valores que aparecen en la matriz se obtienen a partir de la Tabla de Destino a precios básicos. El resto de columnas de esta matriz tienen como valor cero. La integración de los datos en la MDF no es directa, sino a través de la siguiente expresión:

$$
D F=V^{T} \cdot(\widehat{V . e})^{-1} \cdot d
$$

donde $D F$ será la demanda final que se incorpora a la matriz y $d$, los datos procedentes de la Tabla de Destino.

\subsection{Matriz de Cierre (MC)}

Finalmente, la MC se completa a partir del Marco I-O y de los cuadros contables que para los años 2000-2008 publica el INE.

Los hogares perciben su renta por la remuneración de los factores productivos que poseen y por las transferencias que se reciben de los sectores institucionales y del Sector Exterior. Las rentas del trabajo (celda 29/27) se obtienen de las cuentas del total de la economía y de los sectores institucionales (CTESI) de la Contabilidad Nacional, siendo los sueldos y salarios que aparecen en los recursos de los hogares del apartado Cuenta de asignación de la renta primaria (II.1.2). De la misma tabla de destino obtenemos las rentas del capital (29/28), siendo el total del Excedente Bruto de Explotación/Renta Mixta Bruta, cantidad que puede ser observada en la misma cuenta o apartado que las rentas del trabajo anteriores. Las rentas de la propiedad percibidas por los hogares (29/32) se toman de la Cuenta de asignación primaria de la renta de las CTESI, de la parte de los recursos correspondientes a los hogares.

Los hogares perciben prestaciones sociales (29/35) y otras transferencias corrientes (29/36) procedentes no sólo de las AAPP sino también de otros agentes. El importe de las mismas es tomado de la cuenta de distribución secundaria de la renta (II.2) de las CTESI.

Como recursos de los hogares también hay que contabilizar, las cotizaciones sociales a cargo de los empleadores y las cotizaciones sociales. Dado que la contabilización de éstas es una reasignación de las rentas, como recursos de los hogares se toman las cotizaciones sociales a cargo de los empleadores (29/37) que aparecen en la cuenta de asignación de la renta primaria y las cotizaciones sociales (29/38) de la cuenta de distribución secundaria de la renta de las CTESI, en el apartado de recursos de estos agentes. El uso que los hogares hacen de su renta es para el consumo de bienes y servicios y otros gastos. La parte de renta no gastada corresponde al ahorro. 
Los hogares pagan rentas de la propiedad (34/29) correspondientes a los intereses que generan sus pasivos financieros y al pago de las rentas de la tierra. Dicho importe se obtiene de los empleos de la cuenta de asignación de la renta primaria. Otro gasto importante son las transferencias, incluyéndose en éstas las primas netas de seguro no vida, las transferencias corrientes a las ISFLSH o entre hogares, loterías y juegos de azar, etc. El importe de las mismas (36/29) se obtiene de los empleos correspondientes de la cuenta de distribución secundaria de la renta de las CTESI.

El dato de impuestos corrientes sobre la renta, patrimonio,... (41/29) se obtiene de las CTESI del apartado distribución secundaria de la renta. Los impuestos indirectos netos de subvenciones (39/29) se obtienen de la Tabla de Destino, del total de impuestos netos sobre los productos que recaen sobre los hogares al realizar el gasto en consumo final. Otros empleos de los hogares son las prestaciones sociales (35/29) y las cotizaciones sociales (38/29) que se obtienen de los empleos correspondientes de la cuenta de distribución secundaria de la renta de las CTESI.

Los hogares, tanto residentes como no residentes, realizan un gasto en consumo fuera de su territorio económico. El registro de este gasto ha de quedar reflejado junto con el resto de gastos. Por un lado hay que contabilizar las compras de no residentes dentro del territorio económico (29/43) y las compras de residentes fuera del territorio económico (43/29), cuyos datos se obtienen de la Tabla de Destino.

El ahorro privado (31/29) se obtiene a partir de la identidad contable de usos de la renta, esto es, el gasto en consumo final de los hogares más los impuestos (incluida las cotizaciones sociales) y el ahorro, debe ser igual a la suma de todas las rentas percibidas (sueldos y salarios, Excedente Bruto de Explotación/Renta Mixta Bruta, Rentas de la propiedad y transferencias). Éste importe también se puede obtener de los empleos de la cuenta de utilización de la renta disponible de las CTESI. Sin embargo, siguiendo el SEC95, es necesario realizar un ajuste para reflejar en el ahorro las variaciones de las reservas actuariales sobre las que los hogares tienen derecho (29/33), que queda reflejado como ajuste por la variación de la participación neta de los hogares en las reservas de los fondos de pensiones en la Cuenta de utilización de la renta disponible (II.4.1) de las CTESI.

Las ISFLSH perciben rentas de capital (31/28), de la propiedad (31/34), otras transferencias corrientes $(30 / 36)$ y cotizaciones sociales $(30 / 38)$. Como empleos de estas rentas se encuentran las rentas de la propiedad (34/30), prestaciones sociales (35/30) y otras transferencias corrientes (36/30) que se obtienen, al igual que los hogares, de la cuenta de asignación de la renta primaria y de distribución de la cuenta secundaria de las CTESI. El ahorro (31/30) de las ISFLSH se obtiene de igual forma que para los hogares. 
Los siguientes agentes económicos a reflejar en la $\mathrm{MC}$ son las sociedades no financieras y las instituciones financieras. Los ingresos proceden del Excedente de explotación bruto $(32 / 28,33 / 28)$ y de las rentas de la propiedad $(32 / 34$, 33/34) que se obtienen de la cuenta de asignación de la renta primaria, en el apartado de recursos. Otros ingresos proceden de las cotizaciones sociales $(32 / 38,33 / 38)$, tanto efectivas como imputadas, y otras transferencias corrientes $(32 / 36,33 / 36)$, donde se recogen las primas netas e indemnizaciones de seguro no de vida satisfechas por los agentes económicos, que están reflejadas en la cuenta de distribución secundaria de la renta de las CTESI. Los empleos que hacen estos agentes son para el pago de rentas de la propiedad $(34 / 32,34 / 33)$, prestaciones sociales $(35 / 32,35 / 33)$, otras transferencias corrientes $(36 / 32$, 36/33) y los impuestos corrientes sobre la renta y otros impuestos (41/32, 41/33). Las rentas de la propiedad se obtienen de la Cuenta de asignación de la renta primeria y las dos restantes de la Cuenta de distribución secundaria de la renta. La diferencia entre los recursos y los empleos nos dará el ahorro (31/32, 31/33). En ella se tiene en cuenta el ajuste por la variación de la participación neta de los hogares en la reservas de los fondos de pensiones.

El siguiente agente económico a reflejar en la MC son las AAPP. Al igual que cualquier agente obtiene una serie de recursos que proceden fundamentalmente de los impuestos y de las cotizaciones sociales, principalmente. Los usos que le da, mayoritariamente, son el consumo público y las transferencias. Los recursos de las AAPP que hay que contabilizar son, por un lado, el EBE (42/28) y las rentas de la propiedad (42/34), que se obtienen de los correspondientes recursos de la cuenta de asignación de la renta primaria; y, por otro, las cotizaciones sociales (42/38) que son pagadas a la Seguridad Social, los impuestos sobre la renta (42/41) y los impuestos indirectos netos de subvenciones sobre los productos (42/39) y otros impuestos netos sobre la producción (42/40). Estos últimos proceden de la cuenta de asignación de la renta primaria y, cotizaciones e impuestos sobre la renta, de la cuenta de distribución secundaria de la renta; todos del apartado de recursos de las AAPP. Finalmente hay que registrar como recurso otras transferencias (42/36), cuyo importe procede de los recursos de las AAPP, en el apartado otras transferencias corrientes de la cuenta de distribución secundaria de la renta.

En cuanto a los empleos que las AAPP hacen de los anteriores recursos se encuentra el gasto de las propias Administraciones, aunque al quedar registrado a precios básicos, no se incluyen los impuestos indirectos que pagan (39/42) que se toman de la Tabla de Destino del Marco I-O. Los tres últimos empleos a registrar son las rentas de la propiedad (34/42), las prestaciones sociales (35/42) y otras transferencias corrientes (36/42) que se obtienen de los empleos correspondientes de la cuenta de asignación de la renta primaria y de la cuenta de distribución secundaria de la renta, respectivamente. Al ser los recursos superiores a los empleos, resulta un ahorro bruto (31/42) cuyo importe es el que apa- 
rece en la cuenta de utilización de la renta disponible de las CTESI.

El último aspecto a contabilizar en la SAMESP07 es el de las operaciones corrientes con el exterior. En ellas, no sólo hay que contabilizar las exportaciones e importaciones de bienes y servicios, sino también las rentas y transferencias, así como los impuestos. Las importaciones y las exportaciones, obtenidas de las Tablas de Origen y Destino, se encuentran valoradas a precios CIF. Sin embargo, a nivel más agregado hay que utilizar el precio $\mathrm{FOB}^{8}$. Por ello, es necesario realizar un ajuste CIF/FOB (43/43) para pasar unos precios a otros, que se obtiene de las Tablas de Origen y Destino del Marco I-O.

Por otro lado, es necesaria la contabilización de los impuestos indirectos sobre los productos netos de subvenciones. Para el caso de las exportaciones (39/43), éstos se obtienen de la Tabla de Destino. Los impuestos netos sobre los productos (43/39) y otros impuestos netos sobre la producción (43/40) aparecen como recurso en la cuenta exterior de rentas primarias y de transferencias corrientes (apartado II) de las Cuentas del sector resto del mundo y sus subsectores (CRM).

Entre las rentas que deben incluirse se encuentran: a) la remuneración de los asalariados residentes por empleadores no residentes, que incluyen los sueldos y salarios (27/43) y las cotizaciones sociales a cargo de los empleadores (37/43) que se obtienen de los empleos de la Cuenta exterior de rentas primarias de las $\mathrm{CRM}$;, $\mathrm{b}$ ), la remuneración de los asalariados no residentes por empleadores residentes, desglosada en sueldos y salarios (43/27) y cotizaciones sociales a cargo de los empleadores (43/37), que se obtiene de los recursos de la misma cuenta.

De los recursos de la cuenta exterior de rentas primarias se obtienen las rentas de la propiedad (43/34), los impuestos corrientes sobre la renta (43/41), las cotizaciones sociales (43/38), las prestaciones sociales $(43 / 35)$ y otras transferencias corrientes (43/36) pagadas todas ellas a los no residentes. De los empleos obtenemos los mismos conceptos, pero pagados a residentes: rentas de la propiedad (34/43), impuestos corrientes sobre la renta (41/43), cotizaciones sociales $(38 / 43)$, las prestaciones sociales $(35 / 43)$ y otras transferencias corrientes (36/43). El saldo de las operaciones corrientes (30/39) es el ahorro exterior, que junto con el ahorro privado y el ahorro público son los empleos necesarios para la FBC, con los correspondientes impuestos netos sobre los productos $(39 / 31)$.

\footnotetext{
${ }^{8}$ El precio FOB (del acrónimo inglés, Free on board) comprende el valor de los bienes a precios básicos más los márgenes de distribución, junto con los impuestos indirectos netos de subvenciones.
} 


\section{ANÁLISIS SECTORIAL A TRAVÉS DE LA METODOLOGÍA DE RASMUSSEN}

Los sectores clave según Rasmussen (1956) se pueden identificar mediante el uso de dos indicadores denominados backward linkage o efecto arrastre y forward linkage o efecto difusión. El primero de ellos mide el efecto que tiene un cambio en la demanda final de un sector sobre la producción total de una economía; por el contrario, el efecto difusión nos indicaría el efecto que un cambio en la demanda final de todos los sectores tienen sobre la producción de un sector específico.

\subsection{Metodología}

La metodología está basada en el modelo de Leontief. Para el cálculo de los indicadores se parte de la matriz inversa de Leontief, $M=(I-A)^{-1}$, en la que cada uno de los elementos $m_{i j}$ son los denominados multiplicadores simples de Leontief y representan las cantidades de inputs del sector $i$ necesarias para fabricar una unidad de bien por parte del sector $j$.

Una vez obtenidos los multiplicadores se calculan los multiplicadores agregados por columnas y por filas (Rasmussen, 1956). Así, cada uno de los multiplicadores de cada columna y de cada fila vendrá dado, respectivamente, por:

$$
\begin{array}{ll}
M_{\cdot j}=\sum_{i=1}^{n} m_{i j} & \forall j=1,2, \ldots, n \\
M_{i \cdot}=\sum_{j=1}^{n} m_{i j} & \forall i=1,2, \ldots, n
\end{array}
$$

El primer multiplicador agregado (5) indicaría la necesidades de inputs de cada uno de los diferentes sectores que son necesarios para hacer frente a un incremento de una unidad de bien del sector $j$. Análogamente, el segundo multiplicador agregado (6) indicaría la cantidad total de output que debería producir el sector $i$ si el resto de los sectores desean aumentar su producción en una unidad. Dichos multiplicadores ofrecen información sobre el efecto arrastre y el efecto difusión que en un determinado sector va a tener los distintos sectores de la economía. Una vez obtenidos los multiplicadores anteriores se está en disposición de calcular los dos indicadores de los sectores clave.

Los indicadores que miden el efecto arrastre o backward linkage (BL) y el efecto difusión o forward linkage (FL) se calculan a partir de las siguientes expresiones:

$$
B L_{. j}=\frac{M_{\cdot j}}{\frac{1}{n} \sum_{j=1}^{n} m_{i j}} \quad F L_{i}=\frac{M_{i} .}{\frac{1}{n} \sum_{i=1}^{n} m_{i j}}
$$

La normalización de estos índices permite realizar comparaciones entre los diferentes sectores. Un valor mayor (menor) que la unidad indica que el sector 
estudiado va a tener un efecto arrastre o difusión superior (inferior) a la media de los sectores económicos.

$\mathrm{Si}$ el sector tiene un efecto arrastre superior a la unidad $\left(\mathrm{BL}_{j}>1\right)$, significa que un cambio en la demanda final del sector $j$ tiene un efecto mayor sobre la producción que lo que la media de los sectores económicos tienen ${ }^{9}$. Si el sector tiene un efecto difusión superior a la unidad $\left(\mathrm{FL}_{i}>1\right)$, significa que el efecto que la demanda final de todos los sectores sobre la producción del sector $i$ es superior a la media, o lo que es lo mismo, el sector $i$ tendrá que incrementar su producción más que otros sectores para atender a un incremento dado de la demanda ${ }^{10}$.

Sin embargo, estos índices son promedios lo que implica que sería posible que dos sectores tengan los mismos valores de FL o BL aunque tengan distinto efecto sobre la economía. Podría darse el caso de que uno de ellos concentrase su efecto sobre uno o varios sectores pero con un efecto mínimo en el resto; mientras que por el contrario, el otro sector, con el mismo valor podría tener un efecto disperso sobre la totalidad de los sectores aunque con efectos menores sobre cada uno de ellos. Para ello, Rasmussen (1956) define dos nuevos estimadores que medirían la "desviación estándar" de los indicadores BL y FL:

$$
D_{\cdot j}=\frac{\sqrt{\frac{l}{n-1} \sum_{i=1}^{n}\left(m_{i j}-\frac{1}{n} \sum_{i=1}^{n} m_{i j}\right)^{2}}}{\frac{l}{n} \sum_{j=1}^{n} m_{i j}} \quad \forall j=1,2, \ldots, n
$$

y

$$
D_{i}=\frac{\sqrt{\frac{1}{n-1} \sum_{j=1}^{n}\left(m_{i j}-\frac{1}{n} \sum_{j=1}^{n} m_{i j}\right)^{2}}}{\frac{1}{n} \sum_{i=1}^{n} m_{i j}} \quad \forall i=1,2, \ldots, n
$$

La interpretación de estos dos nuevos estimadores refleja la mayor o menor dispersión de los efectos arrastre y difusión sobre el conjunto de sectores. Un valor bajo de $D{ }_{\cdot j}$ reflejaría que el cambio en la demanda final del sector $j$ sobre la producción total de la economía se reparte de manera relativamente uniforme por el conjunto de sectores. Un valor alto significaría que el cambio en la demanda final del sector $j$ se concentra en determinados sectores, influyendo poco en el resto. El índice $D_{i}$. informa de la mayor o menor dispersión del efecto que la demanda final de todos los sectores tiene sobre la producción del sector $i$. Un valor bajo indicaría que todos los sectores afectarían de manera uniforme en la producción del sector $i$. Por el contrario, un valor elevado indicaría que la demanda de algunos sectores serían los que influirían en la producción del sector $i$,

\footnotetext{
${ }^{9}$ Este índice es a lo que Rasmussen llamaba “índice del poder de dispersión”.

${ }^{10}$ Siguiendo a Rasmussen podía ser considerado un "indice de la sensibilidad de la dispersión".
} 
mientras que el resto de los sectores tendrían escasa influencia.

Por otro lado, la identificación de los sectores clave puede hacerse también a partir de los multiplicadores extendidos obtenidos a partir de la MCS. Estos multiplicadores se obtienen de la misma manera que los multiplicadores simples de Leontief pero endogenizando los cuentas de Trabajo (27), Capital (28) y Consumo (29), con lo que se obtiene una matriz de 29 x 29. Los multiplicadores extendidos ofrecen una mayor información que los multiplicadores simples ya que no sólo incluyen las relaciones intersectoriales, sino también el resto de las relaciones que se dan entre los diferentes agentes en una economía. Para el cálculo de los backward linkages y forward linkages se procede de igual forma que anteriormente. Se parte de una matriz cuadrada $X$ de orden $n x n$, donde cada fila y cada columna definen una cuenta económica pero con la diferencia de que $n$ corresponde a la suma de todos los sectores productivos más el resto de las cuentas que se han endogeneizado.

\subsection{Resultados}

La Tabla A.1 muestra los indicadores BL y FL de los 26 sectores productivos obtenidos a partir de los multiplicadores simples de Leontief. Para interpretar la tabla, tomemos como ejemplo el sector Refino de petróleo (6). El valor de $\mathrm{BL}=1.2754$, significa que la demanda final de este sector tiene un efecto en el conjunto de los sectores económicos un $27.54 \%$ superior a la media. Es el denominado efecto arrastre de dicho sector. Por otro lado, su valor de FL = 1.0403 indica que el efecto de la demanda final de todos los sectores sobre el sector Refino del petróleo es un 4.03 \% superior a la media. Dado que ambos valores son superiores a la unidad, puede considerarse este sector un sector clave de la economía española.

Con respecto a los estimadores de dispersión, un alto valor de $D_{._{j}}$ como es el caso del Otros servicios (24) indica que el efecto de la demanda final de este sector se reparte de manera poco uniforme por el conjunto de los sectores, o lo que es lo mismo, el efecto se concentra sobre uno o varios sectores. El sector Pesca (2) presenta un valor alto de $D_{i}$. indicando que el efecto que la demanda final de todos los sectores tiene sobre la producción del sector no es uniforme, o lo que es lo mismo, el sector pesquero es suministrador importante de uno o pocos sectores.

Además del sector Refino de petróleo, otros sectores clave de la economía española son Electricidad (7), Alimentación, bebidas y tabaco (10), Industria química (13), Metalurgia (15), Elaborados metálicos (16), Maquinaria 17), Otras manufacturas (20) y Construcción (21), como se puede observar en la Figura 1. No obstante, en el caso el sector de la Construcción hay que matizar que el efecto arrastre y difusión no se extiende de una manera uniforme por el conjunto de sectores, sino que concentra su efecto en un número limitado de 
ellos, tal y como señalan los elevados valores de dispersión de la Tabla A.1. Como se puede comprobar por los valores más elevados de los multiplicadores simples que aparecen en la tabla original ${ }^{11}$, el sector de la Construcción (21) concentra principalmente su efecto arrastre sobre el propio sector, Otros servicios (24) y Materiales de Construcción (14). Por otro lado, su efecto difusión es debido principalmente al propio sector y a los Servicios destinados a la venta (25).

Figura 1

Clasificación de los sectores clave a partir de los multiplicadores simples

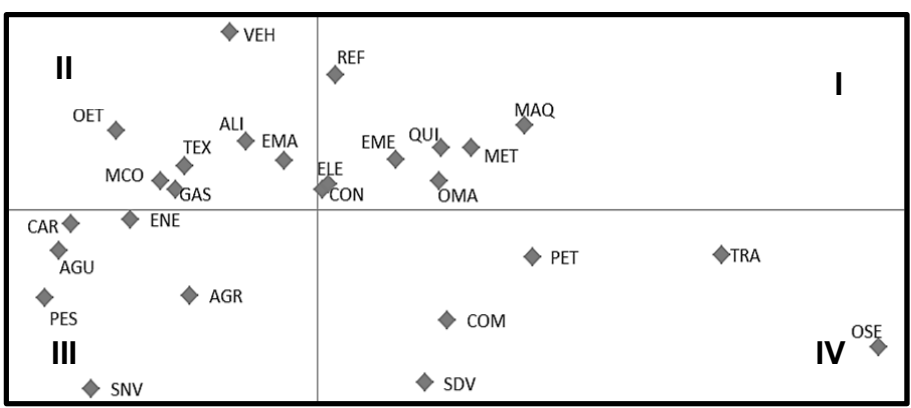

Fuente: Elaboración propia.

En la economía española, además de existir sectores clave, existen sectores con un importante efecto arrastre, identificándose por tener un valor de BL superior a 1 y un valor de BL próximo a 1 . En este caso podemos destacar el sector Vehículos (18), Alimentación, bebidas y tabaco (10) y Elaborados de madera (12). En la región IV de la Figura 1 también se puede ver los sectores de gran efecto difusión. Es el caso de Transportes y comunicaciones (23) y Petróleo y gas natural (24), presentando unos valores FL superiores a 1 y unos valores BL superiores a 0.90 . Por último, los sectores menos relevantes de la economía se encuentran en el sector III, con poco efecto arrastre y difusión, como es el caso Servicios no destinados a la venta (26), Pesca (2), Agricultura, ganadería y silvicultura (1), Carbón (3), Extractivas no energéticas (5) y Agua (9).

Respecto a la dispersión que tienen estos efectos, los sectores con un efecto difusión menos uniformemente repartido son los de Pesca (2), Agua (9) y Carbón (3), con unos valores de $D_{i}$. más elevados, lo que indica que estos sectores son abastecedores principales de unos pocos sectores. En el caso de la Pesca (2), abastece principalmente al sector Alimentación, bebidas y tabaco (10); en el caso del sector Agua (9), al sector agrícola (1); y el sector Carbón (3) abastece

\footnotetext{
${ }^{11}$ La tabla mencionada y el resto de tablas están a disposición de aquellos que las soliciten.
} 
al sector eléctrico (7). En el otro extremo, nos encontraríamos con sectores donde su efecto difusión es bastante uniforme por el conjunto de la economía; como es el caso de Otros servicios (24), Transportes y comunicaciones (23), Comercio y restauración (22) y Servicios destinados a la venta (25).

Asimismo, también es posible medir el grado de dispersión del efecto arrastre que un sector ejerce sobre otros. Los sectores Otros servicios (24), Servicios destinados a la venta (25), Servicios no destinados a la venta (26), Transportes y comunicaciones (23) y Comercio y restauración (22) presentan unos elevados valores de $D_{\bullet j}$, lo que significa que estos sectores concentran su demanda en unos pocos sectores.

Si se analizan los sectores clave de la economía española una vez endogeneizadas las cuentas de Trabajo (27), Capital (28) y Consumo (29), los resultados varían respecto de los anteriores, tal y como se observa en la Tabla A.2.

En este caso, no aparecen sectores clave. Sin embargo, aparecen sectores con un importante efecto arrastre como Alimentación, bebidas y tabaco (10), Metalurgia (15), Maquinaria (17) y Otras manufacturas (20), y sectores con importante efecto difusión como Comercio y restauración (22), Otros servicios (24), Servicios destinados a la venta (25) y Transportes y comunicaciones (23), teniendo un valor FL superior a $1 \mathrm{y}$ un valor BL superior a 0.80 . Por otro lado, aparecen un gran número de sectores poco relevantes. La causa de esta modificación hay que buscarla en la importancia que las cuentas consideradas endógenas tienen en la economía al introducirlas en el modelo, de modo que sectores que antes tenían un gran impacto ven disminuir su importancia sobre el resto de la economía. Con respecto a la dispersión de los efectos, los resultados son muy similares a los que aparecían cuando no estaban endogeneizadas las cuentas, aunque con unos valores inferiores (Tabla A.2).

\section{ANÁLISIS SECTORIAL A TRAVÉS DEL MÉTODO DE EXTRACCIÓN HIPOTÉTICA}

Con esta metodología, la importancia de un sector se deduce a partir de las consecuencias que tendría para la economía su eliminación ${ }^{12}$. El procedimiento consiste en calcular cuál sería la reducción de la producción total que tendría lugar en la economía al suprimir un determinado sector. Cuanto mayor sea la

${ }^{12}$ Este método fue propuesto inicialmente por Paelinck et al. (1965) y, posteriormente, por Strassert (1968), Schultz (1977), Cella (1984), Clements (1990), Dietzenbacher et al. (1993) y Dietzenbacher y van der Linden (1997), entre otros, aunque con propuestas que difieren unas de otras. Así, por ejemplo, Strassert (1968) calcula el eslabonamiento total de los sectores, pero no los separa en backward linkage y forward linkage; Cella (1984) identifica la vinculación total de los sectores y, a partir de ésta, identifica sus dos componentes backward linkage y forward linkage; Dietzenbacher et al. (1993, 1997), por el contrario, calculan los dos componentes de manera independiente. 
disminución del output, mayor será la importancia del sector. La mayor o menor importancia del sector se calculará en términos de efecto arrastre y efecto difusión. El primero de ellos mide el efecto que tiene la eliminación de un sector sobre la producción total; por el contrario, el efecto difusión indicaría el efecto, en términos de coste de oportunidad, que sobre la producción de un sector que ha sido eliminado, tiene la demanda final del resto de los sectores.

\subsection{Metodología}

La metodología que se seguirá para la detección de los sectores clave por el Método de Extracción Hipotética es la de Dietzenbacher et al. (1993), que está basada a su vez en la metodología propuesta por Strassert (1968). Consiste en calcular los efectos sobre la producción derivados de la eliminación de un sector concreto de la economía de un país o región. Dado que se producen dos efectos, se procederá a explicar primero la metodología del efecto arrastre $y$, posteriormente, la del efecto difusión.

Para ello se parte de la ecuación fundamental del Modelo I-O que indica que la producción de cada sector depende de la demanda final:

$$
X=(I-A)^{-1} \cdot D
$$

donde $(I-A)^{-1}$ es la matriz inversa de Leontief, $X$ la producción total y $D$ la demanda final. Consideremos, por ejemplo, que se elimina el sector 1, esto es, el sector 1 no compra inputs intermedios a ningún sector productivo. En este caso, la estructura tecnológica de la economía se mantiene, salvo que los inputs necesarios del sector 1 para el resto de los sectores provienen de la importación. Entonces, los elementos de la matriz A correspondiente a la primera columna serían:

$$
a_{i 1}(-1)=\overline{a_{i 1}}=\frac{z_{i 1}}{x_{1}} 0 \quad \forall i=1,2, \ldots, n
$$

donde $z_{i l}$ es el consumo de input que el sector $i$ hace del sector 1 . En este caso la producción de la economía se habrá reducido, obteniéndose una producción total menor, que será:

$$
X=(I-\bar{A})^{-1} \cdot \bar{D}
$$

donde ${ }^{-}$indica el conjunto de sectores menos el sector eliminado. La eliminación del sector 1 habrá provocado una reducción de la producción que vendrá determinada por:

$$
X-\bar{X}=(I-A)^{-1} \cdot D-(I-\bar{A})^{-1} \cdot \bar{D}
$$

Por otro lado, podemos calcular el impacto que el sector 1 provoca sobre el mismo como

$$
X_{1}-X^{11}=X_{1}-\left(1-a_{11}\right)^{-1} \cdot D_{1}
$$


Así pues, la eliminación de un determinado sector $j$ de la economía habrá producido una reducción de la producción total que vendrá dada por la siguiente expresión:

$$
X-\bar{X}=\left\{\left[\begin{array}{ll}
L_{n}^{j j} & L_{n}^{j r} \\
L_{n}^{r j} & L_{n}^{r r}
\end{array}\right]-\left[\begin{array}{cc}
\left(I-A_{n}^{j j}\right)^{-1} & 0 \\
0 & \left(I-A_{n}^{r r}\right)^{-1}
\end{array}\right]\right\} \cdot\left(\begin{array}{c}
D^{j} \\
D^{r}
\end{array}\right)
$$

donde los superíndices $j$ y $r$ señalan el sector eliminado y el resto de los sectores económicos, respectivamente; y el subíndice $n$, el orden de las matrices que coincidirá con el de los sectores productivos en el caso de que trabajemos con una TIO, o un número superior (sectores más cuentas endógenas), en el caso de una MCS.

Para el cálculo del efecto difusión se procede de igual forma, salvo que en este caso no se utiliza la matriz inversa de Leontief, sino con la matriz inversa Goshiana $G$, el vector de inputs primarios $V$ y la matriz de coeficientes de distribución $B$. La disminución de la producción medida a través del efecto difusión se calculará utilizando la siguiente expresión:

$$
X-\bar{X}=\left(\begin{array}{ll}
V^{j} & V^{r}
\end{array}\right) \cdot\left\{\left[\begin{array}{ll}
G_{n}^{i j} & G_{n}^{i r} \\
G_{n}^{r j} & G_{n}^{r r}
\end{array}\right]-\left[\begin{array}{cc}
\left(I-B_{n}^{i j}\right)^{-1} & 0 \\
0 & \left(I-B_{n}^{r r}\right)^{-1}
\end{array}\right]\right\}
$$

\subsection{Resultados}

En primer lugar analizaremos los sectores clave observando sus backward linkages como puede observarse en la tabla original. La interpretación de los datos se hace en términos de pérdida de output total que tiene la eliminación de un determinado sector. Si se toma la primera celda $(30,596.9)$, éste sería el efecto, expresado en millones de euros, que sobre el output del sector Agricultura, ganadería y silvicultura (1) tiene la eliminación del propio sector; en el caso de la celda 2 de la misma columna (79.1), nos daría el efecto, expresado en millones de euros, que la eliminación del sector agrícola tiene sobre la Pesca (2). La suma de la primera columna coincidiría con el efecto, que en términos de producción sobre la economía, tendría la eliminación del sector Agrícola (1).

La Tabla 2 muestra el total de cada una de las columnas. En ella se puede observar que para los backward linkages, los mayores efectos sobre el conjunto de la economía corresponden a los sectores Comercio y restauración (22), Construcción (21), Otros servicios (24) y Transportes y Comunicaciones (23). Por otro lado, los sectores que menos relevancia tienen en la economía española son Carbón (3), Pesca (2) Agua (9).

Adicionalmente, a partir de estos datos puede observarse la dependencia de los sectores. Así, se aprecia que la hipotética desaparición del sector Refino de petróleo (6) o Gas (8) no sólo produce una importante disminución en la producción del propio sector, sino también en la del sector Petróleo y gas natural 
(4), como se aprecia en la tabla original. Esto mismo se puede observar en el sector Alimentación, bebidas y tabaco (10) respecto del sector Agrícola (1), o del sector Vehículos (18) y Otros elementos de transporte (19) respecto del sector Metalúrgico (15). Hay, por el contrario, sectores donde su influencia se extiende por un mayor número de ellos, como son los casos de Servicios no destinados a la venta (26) o Vehículos (18).

Tabla 2

Sectores clave a partir del método de extracción sin cuentas endógenas (en mill. €)

\begin{tabular}{|l|c|c|}
\hline \multicolumn{1}{|c|}{ Sector (*) } & Backward Linkage & Forward Linkage \\
\hline 1. Agricultura, ganadería y silvicultura & $71,023.1$ & $72,757.1$ \\
\hline 2. Pesca & $4,908.4$ & $4,046.8$ \\
\hline 3. Carbón & $4,022.3$ & $9,108.7$ \\
\hline 4. Petróleo y gas natural & $29,170.7$ & $96,831.6$ \\
\hline 5. Extractivas no energéticas & $16,127.0$ & $32,362.5$ \\
\hline 6. Refino de petróleo & $80,036.9$ & $90,331.1$ \\
\hline 7. Energía eléctrica & $76,771.2$ & $85,298.5$ \\
\hline 8. Gas & $26,828.7$ & $40,901.6$ \\
\hline 9. Agua & $11,957.5$ & $10,947.1$ \\
\hline 10. Alimentación, bebidas y tabaco & $147,417.1$ & $116,636.1$ \\
\hline 11. Textil y piel & $29,616.2$ & $27,800.8$ \\
\hline 12. Elaborados de madera & $50,189.5$ & $62,452.0$ \\
\hline 13. Industria química & $89,094.5$ & $101,522.3$ \\
\hline 14. Materiales de construcción & $87,296.3$ & $84,347.6$ \\
\hline 15. Metalurgia & $106,237.1$ & $146,565.9$ \\
\hline 16. Elaborados metálicos & $111,283.4$ & $130,135.1$ \\
\hline 17. Maquinaria & $128,116.4$ & $134,377.7$ \\
\hline 18. Vehículos & $83,124.5$ & $61,065.0$ \\
\hline 19. Otros elementos de transporte & $24,649.2$ & $18,050.6$ \\
\hline 20. Otras manufacturas & $137,681.4$ & $157,604.2$ \\
\hline 21. Construcción & $293,463.2$ & $211,078.9$ \\
\hline 22. Comercio y restauración & $311,654.4$ & $249,397.2$ \\
\hline 23. Transportes y comunicaciones & $200,191.2$ & $224,155.2$ \\
\hline 24. Otros servicios & $231,538.3$ & $294,427.4$ \\
\hline 25. Servicios destinados a la venta & $184,222.8$ & $193,614.2$ \\
\hline 26. Servicios no destinados a la venta & $131,431.9$ & $72,833.7$ \\
\hline
\end{tabular}

(*) Los mayores valores aparecen sombreados

Fuente: Elaboración propia.

Respecto al efecto difusión los resultados se interpretan como el impacto que, sobre el sector eliminado, tiene la demanda de otros sectores en términos 
de coste de oportunidad. Así, si se observa la tabla original, la primera celda $(18,040.7)$, indicaría la caída en la producción, en millones de euros, del sector Agrícola (1) que provocaría la desaparición del propio sector; en el caso de la segunda celda (73.8), esta cantidad sería la caída en la producción del sector Agrícola que, en términos de coste de oportunidad, tendría su desaparición debido al no poder suministrar inputs para abastecer la demanda del sector Pesca (2). La suma de la primera columna, daría la caída en la producción del sector Agricultura, ganadería y silvicultura (1) que, en términos de coste de oportunidad, tiene su desaparición dado que la demanda del resto de sectores no puede ser suministrada.

Tabla 3

Sectores clave a partir del método de extracción con cuentas endógenas (en mill. €)

\begin{tabular}{|l|c|c|}
\hline \multicolumn{1}{|c|}{ Sector ( $^{*}$ ) } & Backward Linkage & Forward Linkage \\
\hline 1. Agricultura, ganadería y silvicultura & $127,453.6$ & $168,701.9$ \\
\hline 2. Pesca & $11,183.3$ & $13,361.2$ \\
\hline 3. Carbón & $5,332.0$ & $12,876.4$ \\
\hline 4. Petróleo y gas natural & $29,528.8$ & $138,277.3$ \\
\hline 5. Extractivas no energéticas & $21,970.0$ & $41,615.3$ \\
\hline 6. Refino de petróleo & $118,850.1$ & $135,407.7$ \\
\hline 7. Energía eléctrica & $117,533.2$ & $143,255.2$ \\
\hline 8. Gas & $39,872.9$ & $60,927.2$ \\
\hline 9. Agua & $21,716.4$ & $22,709.1$ \\
\hline 10. Alimentación, bebidas y tabaco & $272,241.8$ & $252,418.0$ \\
\hline 11. Textil y piel & $69,934.2$ & $74,070.7$ \\
\hline 12. Elaborados de madera & $71,852.1$ & $93,787.0$ \\
\hline 13. Industria química & $135,271.1$ & $160,398.8$ \\
\hline 14. Materiales de construcción & $125,445.0$ & $112,540.1$ \\
\hline 15. Metalurgia & $141,159.9$ & $194,227.7$ \\
\hline 16. Elaborados metálicos & $158,447.6$ & $181,334.4$ \\
\hline 17. Maquinaria & $192,201.2$ & $207,102.7$ \\
\hline 18. Vehículos & $149,440.6$ & $120,850.3$ \\
\hline 19. Otros elementos de transporte & $39,996.3$ & $29,364.0$ \\
\hline 20. Otras manufacturas & $208,749.7$ & $244,335.1$ \\
\hline 21. Construcción & $534,433.6$ & $403,097.6$ \\
\hline 22. Comercio y restauración & $689,069.8$ & $699,011.4$ \\
\hline 23. Transportes y comunicaciones & $349,220.7$ & $423,154.3$ \\
\hline 24. Otros servicios & $421,408.8$ & $566,303.5$ \\
\hline 25. Servicios destinados a la venta & $465,750.8$ & $572,431.0$ \\
\hline 26. Servicios no destinados a la venta & $386,470.7$ & $218,275.5$ \\
\hline
\end{tabular}

$\left(^{*}\right)$ Los mayores valores aparecen sombreados

Fuente: Elaboración propia. 
La Tabla 2 muestra que los sectores que tienen unos mayores efectos difusión son Otros servicios (24), Comercio y restauración (22), Transportes y Comunicaciones (23) y Construcción (21). En concreto, son los mismos que presentaban un mayor efecto arrastre. Por ello, puede decirse que se trata de sectores clave de la economía española. En el otro extremo nos encontramos con aquellos sectores menos relevantes como es el caso de Pesca (2), Carbón (3), Agua (9) y Otros elementos de transporte (19). Al igual que con los $b a$ ckward linkages, en este caso se puede ver la dependencia de unos sectores y otros. Por ejemplo, en el caso del sector Agrícola (1) únala mayor reducción de su producción mayor sería generada por la demanda del sector Alimentación, bebidas y tabaco (10); en el caso del sector Pesca (2), sería el sector alimentario y el sector Comercio y restauración (22); y así, en el resto de los sectores. Otros sectores que también tienen una importancia destacable son Metalurgia (15), Maquinaria (17), Otras manufacturas (20) y Servicios destinados a la venta (25).

La endogenización de cuentas, tal y como puede observarse en la Tabla 3, hace que el efecto de la eliminación de un determinado sector sea mucho más intensa, apreciándose por unos mayores valores en el caso del efecto arrastre y menores en el caso del efecto difusión, pero produce pocas modificaciones en la determinación de sectores clave de la economía, así como en el resto de sectores.

\section{ANÁLISIS SECTORIAL A PARTIR DE LA MATRIZ DEL PRODUCTO MULTIPLICADOR}

La tercera metodología utilizada es la que se deriva del cálculo de la MPM. Esta se obtiene a partir de la matriz inversa de Leontief o, en el caso de considerar las cuentas endógenas, a partir de los multiplicadores contables. Esta metodología tiene en cuenta, de manera conjunta, el efecto arrastre y el efecto difusión, esto es, el efecto que los cambios en la demanda de un sector tiene sobre el conjunto de la economía y el efecto que un cambio en la demanda del conjunto de sectores tiene sobre un sector determinado, respectivamente.

\subsection{Metodología}

Para el cálculo de la MPM se parte de la matriz inversa de Leontief, $M=(I-$ $A)^{-1}$ (Sonis et al. 1997). Una vez obtenida, se calculan los vectores de multiplicadores $\left(B_{\bullet j}, B_{i} \cdot\right)$, en los que cada uno de los elementos del par se calcula sumando los multiplicadores simples de cada columna y de cada fila, respectivamente:

$$
B_{. j}=\sum_{i=1}^{n} m_{i j} \quad \forall j=1,2, \ldots, n \quad B_{i}=\sum_{j=1}^{n} m_{i j} \quad \forall i=1,2, \ldots, n
$$

siendo $m_{i j}$, los elementos de la matriz inversa de Leontief. Una vez obtenidos estos vectores de multiplicadores se calcula cada uno de los elementos de la 
MPM como el producto de cada par, corregidos por un factor que denominamos "intensidad global" que no es más que la suma de todos los elementos de la matriz inversa de Leontief $(V)$ :

$$
M P M=\frac{1}{V} / / B_{. j} \quad B_{i \cdot} / / \quad \forall i, j=1,2, \ldots, n
$$

donde

$$
V=\sum_{i=1}^{n} \sum_{j=1}^{n} m_{i j}
$$

\subsection{Resultados}

La MPM permite analizar la interdependencia que existe entre los diferentes sectores productivos de la economía española, así como la mayor o menor importancia de los mismos como demandantes o como suministradores de inputs. Los datos de la MPM que aparecen en la tabla original se han condensado en la Tabla 4. En ella se puede ver la suma total por columnas y por filas de la MPM. La suma por columnas nos dará la importancia que tienen los diferentes sectores como demandantes de inputs, esto es, el efecto arrastre de los mismos. La suma de filas nos indica la importancia de los sectores como suministradores de inputs; el efecto difusión de los sectores.

La Tabla 4 muestra que los mayores valores para el efecto arrastre aparecen en los sectores Vehículos (18), Refino de petróleo (6), Metalurgia (15), Otros elementos de transporte (19) y Alimentación, bebidas y tabaco (10). En el caso del efecto difusión resulta que los mayores valores son para el sector Otros servicios (24), Transportes y Comunicaciones (23), Petróleo y gas natural (4), Metalurgia (15) y Maquinaria (17). Por el contrario, los sectores Servicios no destinados a la venta (26), Servicios destinados a la venta (25), Otros servicios (24), Comercio y restauración (22) y Pesca (2) son los que presentan menores valores para el efecto arrastre, mientras que los sectores Pesca (2), Agua (9), Carbón (3), Servicios no destinados a la venta (26) y Otros elementos de transporte (19) son los que muestran menores valores para el efecto difusión.

Los sectores con valores superiores a la media, tanto para el efecto arrastre como para el efecto difusión corresponden a los sectores Metalurgia (15), Maquinaria (17), Otras manufacturas (20), Química (13), Elaborados metálicos (16), Refino de petróleo (6), Energía eléctrica (7) y Construcción (21). Por el contrario, los sectores menos relevantes, con valores de efecto arrastre y difusión por debajo de la media corresponden Servicios no destinados a la venta (26), Pesca (2) o Agricultura, ganadería y silvicultura (1), entre otros. Los sectores relevantes para el efecto arrastre pero con poco efecto difusión son Elaborados de madera (12), Alimentación, bebidas y tabaco (10) y Vehículos (18) y, por último, los sectores con un importante efecto difusión pero un menor efecto arrastre, como es el caso del sector Transportes y comunicaciones (23) o Petróleo y gas natural (4). 
Tabla 4

Sectores clave a partir de la Matriz del Producto Multiplicador

\begin{tabular}{|c|c|c|}
\hline Sector $\left({ }^{*}\right)$ & Suma de columnas & Suma de filas \\
\hline 1. Agricultura, ganadería y silvicultura & 2.1267 & 1.8516 \\
\hline 2. Pesca & 2.1115 & 1.0263 \\
\hline 3. Carbón & 2.5041 & 1.1749 \\
\hline 4. Petróleo y gas natural & 2.3289 & 3.8042 \\
\hline 5. Extractivas no energéticas & 2.5239 & 1.5151 \\
\hline 6. Refino de petróleo & 3.2870 & 2.6811 \\
\hline 7. Energía eléctrica & 2.7091 & 2.6398 \\
\hline 8. Gas & 2.6818 & 1.7692 \\
\hline 9. Agua & 2.3638 & 1.1069 \\
\hline 10. Alimentación, bebidas y tabaco & 2.9334 & 2.1716 \\
\hline 11. Textil y piel & 2.8037 & 1.8218 \\
\hline 12. Elaborados de madera & 2.8350 & 2.3893 \\
\hline 13. Industria química & 2.9028 & 3.2850 \\
\hline 14. Materiales de construcción & 2.7287 & 1.6827 \\
\hline 15. Metalurgia & 3.0219 & 3.7564 \\
\hline 16. Elaborados metálicos & 2.8421 & 3.0265 \\
\hline 17. Maquinaria & 2.9024 & 3.4537 \\
\hline 18. Vehículos & 3.5102 & 2.0827 \\
\hline 19. Otros elementos de transporte & 2.9911 & 1.4339 \\
\hline 20. Otras manufacturas & 2.7299 & 3.2719 \\
\hline 21. Construcción & 2.6828 & 2.6093 \\
\hline 22. Comercio y restauración & 1.9935 & 3.3192 \\
\hline 23. Transportes y comunicaciones & 2.3409 & 4.8791 \\
\hline 24. Otros servicios & 1.8529 & 5.7745 \\
\hline 25. Servicios destinados a la venta & 1.6661 & 3.1915 \\
\hline 26. Servicios no destinados a la venta & 1.6361 & 1.2924 \\
\hline VALOR MEDIO & 2.5773 & 2.5773 \\
\hline
\end{tabular}

(*) Los valores superiores a la media aparecen sombreados

Fuente: Elaboración propia.

Alternativamente la capacidad de difusión y de arrastre de los sectores se puede apreciar a través de los denominados paisajes tridimensionales (Landscape) (Figura 2). En ella se encuentran ordenados los sectores de la economía española según la importancia de los enlaces intersectoriales. Así, por ejemplo, las mayores relaciones se dan entre el sector Otros servicios (24) con los sectores Vehículos (18) y Refino de petróleo (6); la menor entre el sector Pesca (2) con Servicios no destinados a la venta (26).

La MPM se ha obtenido, al igual que en los apartados anteriores, endogenizando las cuentas de Trabajo (27), Capital (28) y Consumo (29) obteniéndose 
una MCS de 29 x 29. La Tabla 5 incluye un resumen de los resultados, que corresponden con las sumas de columnas y filas.

Figura 2

Landscape de los sectores productivos de la economía española

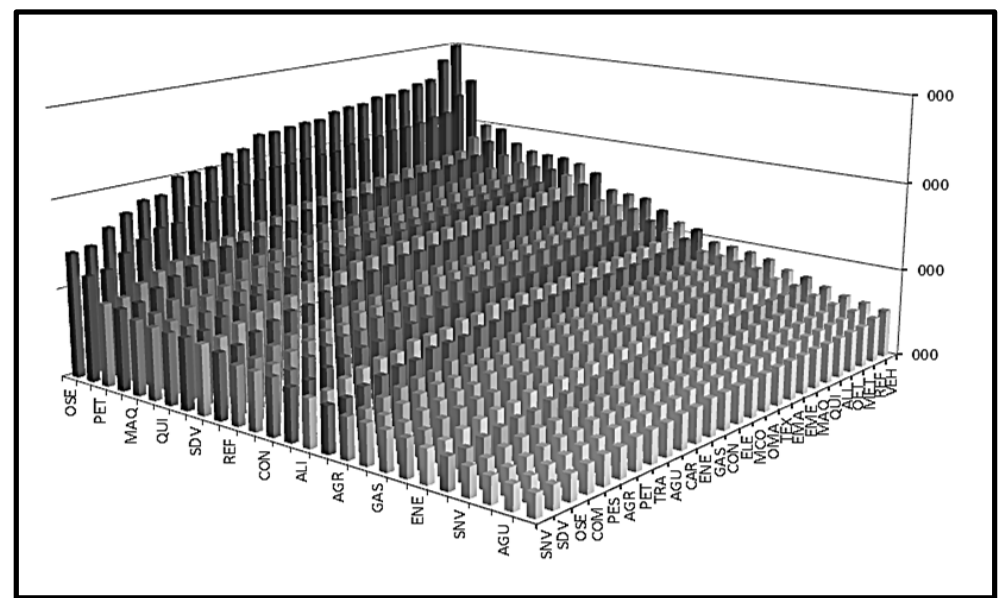

Fuente: Elaboración propia.

A partir de la Tabla 5 se observa que al introducir nuevas cuentas y, por tanto, nuevas relaciones entre los agentes económicos, los valores de la MPM aumentan con un mayor nivel de endogeneización. Por otro lado, se produce una variación en los sectores relevantes. Así, respecto del efecto difusión, si se tiene en cuenta aquellos sectores que tienen un valor mayor que el valor medio, no aparecen el sector Refino de petróleo (6), eléctrico (7) y Elaborados metalicos (16), respecto del caso anterior, cuando no aparecían endogeneizadas las cuentas. Por el contrario, aparecen los sectores Alimentación, bebidas y tabaco (10) y Elaborados de madera (12). Algo similar ocurre con el efecto arrastre; con un valor mayor que la media nos encontramos ahora el sector Carbón (3), y no nos encontramos a sectores como Energía eléctrica (7), Gas (8) y Agua (9).

Si se tiene en cuenta los dos efectos a la vez, aquellos sectores que presentan valores para ambos efectos por encima de la media son los de Alimentación, bebidas y tabaco (10), Química (13), Metalurgia (15), Maquinaria (17), Otras manufacturas (20) y Construcción (21). Por el contrario, los sectores menos relevantes son poco numerosos, y entre ellos podemos ver el sector Servicios no destinados a la venta (26), Agua (9), Pesca (2) y Extractivas no energéticas (5). Como sectores con un importante efecto arrastre aparecen Refino de petróleo (6), Elaborados metálicos (16) y Vehículos (18) y sectores con un importante efecto difusión, Petróleo y gas natural (4), Comercio y restauración (22), Trans- 
portes y comunicaciones (23), Otros servicios (24) y Servicios destinados a la venta (25).

\section{Tabla 5}

Sectores clave a partir de la Matriz del Producto Multiplicador con cuentas endógenas

\begin{tabular}{|c|c|c|}
\hline Sector $\left({ }^{*}\right)$ & Suma de columnas & Suma de filas \\
\hline 1. Agricultura, ganadería y silvicultura & 3.5693 & 2.7776 \\
\hline 2. Pesca & 3.4677 & 1.0561 \\
\hline 3. Carbón & 3.6725 & 1.1360 \\
\hline 4. Petróleo y gas natural & 3.4777 & 4.1750 \\
\hline 5. Extractivas no energéticas & 3.5157 & 1.5097 \\
\hline 6. Refino de petróleo & 3.9885 & 3.2616 \\
\hline 7. Energía eléctrica & 3.6139 & 3.2090 \\
\hline 8. Gas & 3.6282 & 1.8894 \\
\hline 9. Agua & 3.5165 & 1.1536 \\
\hline 10. Alimentación, bebidas y tabaco & 3.9367 & 4.3984 \\
\hline 11. Textil y piel & 3.8203 & 2.4843 \\
\hline 12. Elaborados de madera & 3.8030 & 2.6774 \\
\hline 13. Industria química & 3.8147 & 3.9297 \\
\hline 14. Materiales de construcción & 3.6949 & 1.8366 \\
\hline 15. Metalurgia & 3.8750 & 4.1773 \\
\hline 16. Elaborados metálicos & 3.8183 & 3.4332 \\
\hline 17. Maquinaria & 3.8503 & 4.2383 \\
\hline 18. Vehículos & 4.1906 & 2.9885 \\
\hline 19. Otros elementos de transporte & 3.9372 & 1.4504 \\
\hline 20. Otras manufacturas & 3.7512 & 4.2398 \\
\hline 21. Construcción & 3.6963 & 3.6800 \\
\hline 22. Comercio y restauración & 3.2865 & 9.5676 \\
\hline 23. Transportes y comunicaciones & 3.4276 & 7.3604 \\
\hline 24. Otros servicios & 3.2175 & 8.7924 \\
\hline 25. Servicios destinados a la venta & 2.9428 & 7.5537 \\
\hline 26. Servicios no destinados a la venta & 3.1721 & 1.7089 \\
\hline VALOR MEDIO & 3.6417 & 3.6417 \\
\hline
\end{tabular}

(*) Los valores superiores a la media aparecen sombreados

Fuente: Elaboración propia.

\section{ANÁLISIS COMPARATIVO DE LOS SECTORES CLAVE DE LA ECONOMÍA ESPAÑOLA}

La Tabla 6 presenta un resumen de los sectores clave obtenidos a partir de cada uno de los métodos utilizados, donde se aprecian diferencias y similitudes en los mismos. Los resultados más similares se obtienen al comparar la meto- 
dología de Rasmussen y el método de la MPM. Esto se debe a que en ambos casos el fundamento metodológico son los multiplicadores simples de Leontief o los multiplicadores contables, y el tratamiento metodológico es muy similar. Sin embargo, en el caso del Método de Extracción Hipotética, aunque también se utiliza la matriz inversa de Leontief, el tratamiento es más elaborado.

\section{Tabla 6}

Cuadro comparativo de los sectores clave de la economía española

\begin{tabular}{|c|c|c|c|}
\hline & $\begin{array}{l}\text { Metodología de } \\
\text { Rasmussen }\end{array}$ & Método de Extracción Hipotética & Matriz del Producto Multiplicador \\
\hline $\begin{array}{c}\text { Sin cuentas } \\
\text { endogeneizadas }\end{array}$ & \begin{tabular}{|l} 
Refino de petróleo (6) \\
Energía eléctrica (7) \\
Industria química (13) \\
Metalurgia (15) \\
Elaborados metálicos (16) \\
Maquinaria (17) \\
Otras manufacturas (20) \\
Construcción (21)
\end{tabular} & $\begin{array}{l}\text { Construcción (21) } \\
\text { Comercio y restauración (22) } \\
\text { Transportes y Comunicaciones (23) } \\
\text { Otros servicios (24) } \\
\text { Servicios destinados a la venta (25) }\end{array}$ & $\begin{array}{l}\text { Refino de petróleo (6) } \\
\text { Energía eléctrica (7) } \\
\text { Industria química (13) } \\
\text { Metalurgia (15) } \\
\text { Elaborados metálicos (16) } \\
\text { Maquinaria (17) } \\
\text { Otras manufacturas (20) } \\
\text { Construcción (21) }\end{array}$ \\
\hline $\begin{array}{c}\text { Con } \\
\text { endogeneización } \\
\text { de las cuentas } \\
\text { Trabajo, Capital y } \\
\text { Consumo }\end{array}$ & & $\begin{array}{l}\text { Construcción (21) } \\
\text { Comercio y restauración (22) } \\
\text { Transportes y Comunicaciones (23) } \\
\text { Otros servicios (24) } \\
\text { Servicios destinados a la venta (25) }\end{array}$ & $\begin{array}{l}\text { Alimentación, bebidas y tabaco (10) } \\
\text { Elaborados de madera (12) } \\
\text { Industria química (13) } \\
\text { Metalurgia (15) } \\
\text { Maquinaria (17) } \\
\text { Otras manufacturas (20) } \\
\text { Construcción (21) }\end{array}$ \\
\hline
\end{tabular}

Fuente: Elaboración propia.

La explicación para estas diferencias hay que buscarla, además de en el método utilizado, en la endogeneización o no de las cuentas.

La metodología de Rasmussen (1956), al igual que la de la MPM, obtiene los mismos resultados cuando no existe endogeneización de cuentas. Los sectores clave que aparecen pertenecen al sector energético, industrial y la construcción. Por el contrario, los sectores clave, cuando se utiliza la metodología de Dietzenbacher et al. (1993), pertenecen al sector terciario, además de la construcción. La razón de todo ello es debida a la metodología usada. La metodología de Rasmussen (1956) da lugar a elementos (multiplicadores) obtenidos en términos relativos, esto es, cada multiplicador de un determinado sector se relaciona con la media de los multiplicadores de dicho sector, de ahí, que el valor obtenido se encuentra homogeneizado. Algo similar ocurre con la MPM, en la que los vectores de multiplicadores obtenidos se corrigen por un factor que es la suma de todos los elementos de la Matriz Inversa de Leontief. Por el contrario, con la metodología de Dietzenbacher et al. (1993), los valores obtenidos son datos absolutos, esto es la reducción que tendría lugar en el output total con la desaparición de un determinado sector. Por ello, aquellos sectores que tengan un mayor valor de producción, tendrán un efecto mayor en la reducción del valor de la misma. Este es el caso de la Construcción y de otras ramas del sector servicios. 
Otra diferencia apreciable es la que se deriva de la comparación de los resultados con cuentas endogeneizadas o no. Con la metodología de Rasmussen (1956), cuando las cuentas de Trabajo (27), Capital (28) y Consumo (29) de los hogares se hacen endógenas, no aparece ningún sector considerado como clave, aunque sí aparecen sectores con un importante efecto arrastre y otros sectores con un importante efecto difusión. Los sectores clave según la metodología de la MPM también sufren variaciones; así, desaparecen sectores como Refino de petróleo (6), Energía eléctrica (7) y Elaborados metálicos (16) y entran como sectores clave Alimentación, bebidas y tabaco (10) y Elaborados de madera (12). Por el contrario, la metodología de Dietzenbacher et al. (1993) utilizada con cuentas endógenas conduce a los mismos resultados que sin cuentas endógenas.

\section{CONCLUSIONES}

La estructura de una MCS es flexible y permite tomar diferentes formas dependiendo de las cuentas que la integren. Para la elaboración de la SAMESP07 se ha tenido en cuenta, además de la agrupación en 26 sectores económicos, utilizando las Tablas de Origen y Destino de la CNE como fuente de datos, el tratamiento que se ha hecho de las mismas. La SAMESP07 parte de la base de que cada producto fabricado tiene su propia estructura de ventas, con independencia de la rama de actividad donde se produjo (Tabla A.1). Este artículo muestra la importancia de una MCS para el análisis económico y, en especial, para el análisis de los sectores clave de la economía española. A través de las interrelaciones que se dan entre los diferentes sectores económicos, se pueden determinar aquellos que tienen una mayor potencialidad para estimular la producción de otros sectores $\mathrm{y}$, por tanto, estimular un rápido crecimiento de la producción total, del empleo y de la renta.

La determinación de los sectores clave se ha realizado a través de tres metodologías diferentes: el método de los sectores clave de Rasmussen (1956), la Metodología de Extracción Hipotética siguiendo a Dietzenbacher et al. (1993) y, mediante la MPM vista a través de Sonis et al. (1997). El uso de una u otra conduce a resultados diferentes. Así, el primero y tercero de estos métodos considera como claves, ramas de actividad del sector energético como Refino de petróleo (6) y Energía eléctrica (7), del sector industrial como Industria química (13), Metalurgia (15), Elaborados metálicos (16), Maquinaria (17), Otras manufacturas (20) y la Construcción (21). En el extremo opuesto, esto es, como sectores poco relevantes de la economía española aparecen Agricultura, ganadería y silvicultura (1), Pesca (2), Agua (9) y Servicios no destinados a la venta (26). Los sectores, considerados como claves con la metodología de Dietzenbacher et al. (1993), son la Construcción (21) y ramas del sector servicios como Comercio y restauración (22), Transportes y comunicaciones (23), Otros servicios (24) 
y Servicios no destinados a la venta (25). Como sectores poco relevantes aparecen Pesca (2), Carbón (3) y Agua (9), entre otros. Así pues, el único nexo en común de las tres metodologías es la consideración de la Construcción (21) como sector clave y Pesca (2) y Agua (9) como poco relevantes.

La diferencias en los resultados al aplicar los distintos métodos derivan del propio tratamiento de los datos. En la metodología de Rasmussen (1956) y de la MPM los datos obtenidos provienen de la homogeneización de los mismos, por el contrario, los valores obtenidos con la metodología de Dietzenbacher et al. (1993) son datos absolutos no corregidos por ningún factor. Con esta metodología, los sectores que tengan un mayor valor de output, tendrán un efecto mayor en la reducción del valor total del mismo, como es el caso de la Construcción (21) y otras ramas del sector terciario.

La endogenización de cuentas ofrece resultados diferentes respecto del análisis a través del modelo de Leontief, donde sólo se tiene en cuenta las cuentas correspondientes a los sectores productivos. Estas diferencias se han concretado en unos mayores valores de los diferentes indicadores utilizados así como de modificaciones en cuanto a sectores clave. Las diferencias son especialmente relevantes en el método de los sectores clave de Rasmussen (1956), donde no aparecen sectores clave, quedando sectores con un importante efecto arrastre $\mathrm{o}$ difusión, como son Alimentación, bebidas y tabaco (10), Comercio y restauración (22), Transportes y comunicaciones (23), Otros servicios (24) y Servicios destinados a la venta (25). También hay algunas diferencias en los sectores clave detectados con la metodología de la MPM. En concreto, tras la endogeneización de cuentas desaparecen sectores como Refino de petróleo (6), Energía eléctrica (7) y Elaborados metálicos (16). Por último, los resultados obtenidos con la metodología de Dietzenbacher et al. (1993) no presentan diferencias tras la endogeneización de las cuentas.

La discrepancia entre los resultados obtenidos según metodologías justifica seguir avanzando en la mejora metodológica aplicada a la identificación de los sectores claves de una economía. En esta línea, investigaciones como la realizada en este artículo podría ser completada utilizando un Modelo de Equilibrio General Aplicado (MEGA), que, a diferencia del modelo lineal usado, no impone tantas restricciones sobre el comportamiento de los agentes y permite formular relaciones de carácter no lineal. 


\section{REFERENCIAS BIBLIOGRÁFICAS}

ALCÁNTARA, V., DEL RÍO, P. y HERNÁNDEZ, F. (2010), "Structural analysis of electricity consumption by productive sectors", Energy, 35(5), 2088-2098.

CÁMARA, A., FLORES, M. y FUENTES, P. (2011), "Análisis económico y medioambiental del sector eléctrico en España”, Estudios de Economía Aplicada, 29(2), 493-514.

CARDENETE, M.A. y MONICHE, L. (2001): "El nuevo marco input-output y la SAM de Andalucía para 1995", Cuadernos de CCEE y EE, 41, 13-31.

CARDENETE, M.A. y LLOP, M. (2005): "Modelos multisectoriales de equilibrio general aplicado en España: Una revisión”, Estudios de Economía Aplicada, 23(2): 385-404.

CARDENETE, M.A. y SANCHO, F. (2006): "Missing Links in Key Sector Analysis", Economic Systems Research, 18, 319-325.

CARDENETE, M.A. y SANCHO, F. (2007): "Elaboración de una matriz de contabilidad social a través del Método de Entropía Cruzada: España 1995", Estadística Española, 48(161), 67-100.

CARDENETE, M.A., LLANES, G., LIMA, C. y MORILLA, C. (2008): "Detection of key sectors by using social accounting matrices: an alternative approach", Journal of Applied Input-Output Analysis, 13, 83-91.

CARDENETE, M.A., FUENTES, P. y POLO, C. (2010): "Análisis de sectores clave a partir de la matriz de contabilidad social de Andalucía para el año 2000", Revista de Estudios Regionales, 88, 15-44.

CELLA, G. (1984) "The Input-Output Measurement of Interindustry Linkages", Oxford Bulletin of Economics and Statistics, 46, 73-84.

CLEMENTS B.J. (1990). "On the Decomposition and Normalization of Interindustry Linkages", Economics Letters 33: 337-340

COMISIÓN EUROPEA (1996), Reglamento 2223/96 del Consejo de 25 de junio de 1996 relativo al Sistema Europeo de Cuentas Nacionales y Regionales de la Comunidad (DO L 310 de 30.11.1996)

DIETZENBACHER, E. y VAN DER LINDEN, J. A. (1997) "Sectoral and spatial linkages in the EC production structure", Journal of Regional Science, 37(2), 235-257.

DIETZENBACHER, E., VAN DER LINDEN, J. A. y STEENGE, A. E. (1993) "The Regional Extraction Method: EC Input-Output Comparisons", Economic Systems Research, 5, 180-206.

DIETZENBACHER, E. ROMERO, I. y BOSMA, N. (2005): "Using Average Propagation Lengths to Identify Production Chains in the Andalusian Economy", Estudios de Economía Aplicada, 23(2): 405-422.

EUROSTAT (2008): Eurostat Manual of Supply, Use and Input-Output Tables, Methodologies and Working Papers, European Communities, Luxembourg 
FERNÁNDEZ, M. y POLO, C. (2001): "Una nueva matriz de contabilidad social para España: la SAM-90”, Estadística Española, 43(148), 281-311.

GUERRA, A.I. y SANCHO, F.. (2010): "Measuring energy linkages with the hypothetical extraction method: An application to Spain", Energy Economics, 32(4), 831-837.

HIRSCHMAN, A. (1958): "The strategy of economic development", New Haven: Yale University Press. Versión española: "La estrategia del desarrollo económico", México, Fondo de Cultura Económica, 1964.

KEHOE, T., MANRESA, A., POLO, C. y SANCHO, F. (1988): "Una Matriz de Contabilidad Social de la economía española", Estadística Española, 30(117), 5-33.

KOP JANSEN, P. y TEN RAA, T. (1990): "The Choice of Model in the Construction of Input-Output Coefficients Matrices", International Economic Review, 31(1), 213-227.

LEONTIEF, W. (1966), "Input-Output Economics", $2^{\mathrm{a}}$ ed., New York, Oxford University Press.

LLANO, C. (2009), "Efectos de desbordamiento interregional en España. Una estimación a través del modelo input-output interregional", Investigaciones Regionales, 16, 181-188.

PAELINCK, J., CAEVEL, J DE y DEGUELDRE, J. (1965) "Analyse Quantitative de Certains Phénomènes du Développement Régional Polarisé: Essai de Simulation Statique d'Itinéraires de Propagation", In: Problèmes de Conversion Economique: Analyses Théoriques et Etudes Appliquées (Ed. Génin M. $T$ ), pp. 341-387, Bibliothèque de l'Institut de Science Economique, Paris.

PYATT, G. y ROE, A. (1977): Social Accounting for development planning with special reference to Sri Lanka, Cambridge University Press.

RASMUSSEN, P. (1956), "Studies in Intersectorial Relations", Copenhagen, Einar Harks. Versión en español: "Relaciones intersectoriales", Madrid, editorial Aguilar, 1963.

ROBLES, L. y SANJUAN, J (2008): "Sectores y clusters claves en la economía española“, ICE, Revista de Economía, 843, 183-257.

RODRÍGUEZ, C. y LLANES, G. (2005): "Estimación anual de matrices de contabilidad social usando el método de minimización de la entropía cruzada: aplicación a la economía española del año 2000“, Estudios de Economía Aplicada, 23 (1), 279-302.

SONIS, M., HEWINGS, G.J.D. y GUO, J. (1997), analysis of China's metropolitan economies: an input-output perspective". In M. Chatterji and Y. Kaizhong (eds), Regional Science in Developing Countries (Basingstoke, Macmillan Press), 147-162.

SCHULTZ, S. (1977): "Approaches to Identifying Key Sectors Empirically by Means of Input-Output Analysis", Journal of Development Studies, 14, 7796. 
STONE, R. (1962): "A social accounting matrix for 1960. A programme for growth", London, Chapman and Hall.

STRASSERT, G. (1968) "Zur Bestimmung Strategischer Sektoren mit Hilfe von Input-Output Modelen", Jahrbücher für Nationalökonomie und Statistik, 182, 211-215.

TEN RAA, T. y RUEDA-CANTUCHE, J.M. (2003): "The Construction of InputOutput Coefficients Matrices in an Axiomatic Context: Some Further Considerations", Economic Systems Research, 15(4), 439-455.

URIEL, E., BENEITO, P., FERRI, J. y MOLTÓ, M.L. (1997): "Matriz de Contabilidad Social de España 1990 (MCS-90)“, Madrid, Instituto Nacional de Estadística. 


\section{ANEXO}

Tabla A.1

Indicadores para la detección de sectores clave a partir de los multiplicadores simples

\begin{tabular}{|c|c|c|c|c|}
\hline Sector $\left({ }^{*}\right)$ & Backward Linkage & Forward Linkage & $D_{. j}$ & $D_{i \cdot}$ \\
\hline 1. Agricultura, ganadería y silvicultura & 0.8252 & 0.7184 & 2.6413 & 3.1315 \\
\hline 2. Pesca & 0.8192 & 0.3982 & 2.3578 & 4.9685 \\
\hline 3. Carbón & 0.9716 & 0.4559 & 2.0025 & 4.3564 \\
\hline 4. Petróleo y gas natural & 0.9036 & 1.4760 & 2.3537 & 1.8854 \\
\hline 5. Extractivas no energéticas & 0.9793 & 0.5878 & 2.0355 & 3.3978 \\
\hline 6. Refino de petróleo & 1.2754 & 1.0403 & 2.2762 & 2.3549 \\
\hline 7. Energía eléctrica & 1.0511 & 1.0243 & 2.3369 & 2.3456 \\
\hline 8. Gas & 1.0405 & 0.6864 & 2.2703 & 2.9799 \\
\hline 9. Agua & 0.9172 & 0.4295 & 2.1447 & 4.6068 \\
\hline 10. Alimentación, bebidas y tabaco & 1.1382 & 0.8426 & 2.3415 & 3.1480 \\
\hline 11. Textil y piel & 1.0878 & 0.7069 & 2.6493 & 4.1177 \\
\hline 12. Elaborados de madera & 1.1000 & 0.9271 & 2.4720 & 2.9406 \\
\hline 13. Industria química & 1.1263 & 1.2746 & 2.4276 & 2.0933 \\
\hline 14. Materiales de construcción & 1.0587 & 0.6529 & 2.1302 & 3.4636 \\
\hline 15. Metalurgia & 1.1725 & 1.4575 & 2.0714 & 1.7416 \\
\hline 16. Elaborados metálicos & 1.1027 & 1.1743 & 2.1549 & 1.8686 \\
\hline 17. Maquinaria & 1.1261 & 1.3400 & 2.3517 & 1.8969 \\
\hline 18. Vehículos & 1.3620 & 0.8081 & 2.4427 & 4.1442 \\
\hline 19. Otros elementos de transporte & 1.1605 & 0.5563 & 2.0474 & 4.2963 \\
\hline 20. Otras manufacturas & 1.0592 & 1.2695 & 2.1912 & 1.7847 \\
\hline 21. Construcción & 1.0409 & 1.0124 & 2.8413 & 2.8966 \\
\hline 22. Comercio y restauración & 0.7735 & 1.2878 & 2.7148 & 1.5322 \\
\hline 23. Transportes y comunicaciones & 0.9083 & 1.8931 & 2.9524 & 1.3247 \\
\hline 24. Otros servicios & 0.7189 & 2.2405 & 3.4271 & 0.9666 \\
\hline 25. Servicios destinados a la venta & 0.6465 & 1.2383 & 3.3011 & 1.6312 \\
\hline 26. Servicios no destinados a la venta & 0.6348 & 0.5014 & 3.0963 & 3.9388 \\
\hline Variación media & & & 2.4628 & 2.8389 \\
\hline
\end{tabular}

(*) Los valores superiores a 1 en los BL y FL, al igual que los valores de D superiores a la media, aparecen sombreados

Fuente: Elaboración propia. 
Tabla A.2

Indicadores para la detección de sectores clave a partir de los multiplicadores extendidos

(con endogenización de las cuentas de Trabajo, Capital y Consumo)

\begin{tabular}{|c|c|c|c|c|}
\hline Sector $\left({ }^{*}\right)$ & Backward Linkage & Forward Linkage & $D_{. j}$ & $D_{i} \cdot$ \\
\hline 1. Agricultura, ganadería y silvicultura & 1.0138 & 0.5103 & 1.6004 & 2.0532 \\
\hline 2. Pesca & 0.9850 & 0.1940 & 1.4437 & 4.7299 \\
\hline 3. Carbón & 1.0431 & 0.2087 & 1.3238 & 4.4163 \\
\hline 4. Petróleo y gas natural & 0.9878 & 0.7670 & 1.4729 & 1.6999 \\
\hline 5. Extractivas no energéticas & 0.9986 & 0.2774 & 1.3540 & 3.3470 \\
\hline 6. Refino de petróleo & 1.1329 & 0.5992 & 1.4649 & 1.9050 \\
\hline 7. Energía eléctrica & 1.0265 & 0.5896 & 1.4846 & 1.8982 \\
\hline 8. Gas & 1.0306 & 0.3471 & 1.4472 & 2.7405 \\
\hline 9. Agua & 0.9988 & 0.2119 & 1.3809 & 4.3310 \\
\hline 10. Alimentación, bebidas y tabaco & 1.1182 & 0.8081 & 1.5101 & 1.5285 \\
\hline 11. Textil y piel & 1.0851 & 0.4564 & 1.5678 & 2.9609 \\
\hline 12. Elaborados de madera & 1.0802 & 0.4919 & 1.5040 & 2.5796 \\
\hline 13. Industria química & 1.0835 & 0.7220 & 1.5017 & 1.7282 \\
\hline 14. Materiales de construcción & 1.0495 & 0.3374 & 1.3806 & 3.1137 \\
\hline 15. Metalurgia & 1.1006 & 0.7675 & 1.3679 & 1.5548 \\
\hline 16. Elaborados metálicos & 1.0845 & 0.6307 & 1.3851 & 1.6325 \\
\hline 17. Maquinaria & 1.0936 & 0.7787 & 1.4654 & 1.5305 \\
\hline 18. Vehículos & 1.1903 & 0.5490 & 1.5378 & 2.8296 \\
\hline 19. Otros elementos de transporte & 1.1183 & 0.2665 & 1.3343 & 4.1635 \\
\hline 20. Otras manufacturas & 1.0655 & 0.7789 & 1.4131 & 1.3657 \\
\hline 21. Construcción & 1.0499 & 0.6761 & 1.6370 & 2.0170 \\
\hline 22. Comercio y restauración & 0.9335 & 1.7578 & 1.6884 & 0.5281 \\
\hline 23. Transportes y comunicaciones & 0.9736 & 1.3522 & 1.7053 & 0.8745 \\
\hline 24. Otros servicios & 0.9139 & 1.6153 & 1.7846 & 0.6460 \\
\hline 25. Servicios destinados a la venta & 0.8359 & 1.3878 & 1.8074 & 0.6694 \\
\hline 26. Servicios no destinados a la venta & 0.9010 & 0.3140 & 1.6119 & 2.9245 \\
\hline Variación media & & & 1.5067 & 2.2988 \\
\hline
\end{tabular}

$\left(^{*}\right)$ Los valores superiores a 1 en los BL y FL, al igual que los valores de D superiores a la media, aparecen sombreados

Fuente: Elaboración propia. 
